Cite this: Phys. Chem. Chem. Phys., 2013

\title{
Molecular basis for solvent dependent morphologies observed on electrosprayed surfaces $\dagger$
}

15, 17862

Received 22nd July 2013,

Accepted 27th August 2013

DOI: $10.1039 / c 3 c p 53079 e$

www.rsc.org/pccp

\author{
Elif Ozden-Yenigun, ${ }^{a}$ Eren Simsek, ${ }^{\text {ab }}$ Yusuf Ziya Menceloglu ${ }^{a}$ and Canan Atilgan ${ }^{\star a}$
}

\begin{abstract}
We study the causes of the observed tunable hydrophobicity of poly(styrene-co-perfluoroalkyl ethylacrylate) electrosprayed in THF, DMF, and THF:DMF (1:1) solvents. Under the assumption that equilibrium morphologies in the solvent significantly affect the patterns observed on electrosprayed surfaces, we use atomistic and coarse-grained simulations supported by dynamic light scattering (DLS) experiments to focus on the parameters that affect the resulting morphology of superhydrophobic electrosprayed beads. The differing equilibrium chain size distributions in these solvents examined by DLS are corroborated by chain dimensions obtained via molecular dynamics simulations. Mesoscopic morphologies monitored by dissipative particle dynamics simulations explain experimental observations; in particular, the preference of the polymer for THF over DMF in the binary mixture rationalizes the dual scale roughness driven by stable microphase separation. Drying phenomena that affect resultant dual-scale roughness are described in three stages, each interpreted by concentration dependent diffusion and surface mass transfer coefficients of the solvents. Irrespective of the presence of polar groups in the structure, a conflict between the lower-boiling point solvent adhering to the polymer and the less volatile solvent abundant in the bulk leads to perfectly hydrophobic surfaces.
\end{abstract}

\section{Introduction}

Fluorinated materials have attracted considerable attention due to their low surface energy, ${ }^{1}$ corrosion resistance, ${ }^{2}$ thermal stability, ${ }^{3,4}$ and low refractive index. ${ }^{5}$ Particularly, fluorocarbons have found numerous uses as hydrophobic coatings for low humidity and adhesion applications due to the nonpolar nature of the $-\mathrm{CF}_{x}$ groups. ${ }^{6}$ Homopolymers composed of perfluorinated chains or pendant groups are preferred under high temperature or rigorous chemical conditions, but their low or non-solubility in common solvents limit their use in many applications. However, for surface applications focusing on hydrophobicity, copolymers of fluorinated and conventional monomers can be effectively employed since the fluorinated groups segregate at the solid-air interface to decrease surface energy. ${ }^{1,7-9}$ In addition, self-assembly of fluorinated block copolymers in various environments has been successfully utilized to fabricate nano-structures having a wide range of morphologies which have found applications in emerging

\footnotetext{
${ }^{a}$ Sabancı University, Faculty of Engineering and Natural Sciences, Material Science and Engineering Program, Istanbul, Turkey. E-mail: canan@sabanciuniv.edu; Fax: +90 (216) 48395 50; Tel: +90 (216) 4839523

${ }^{b}$ Ak-Kim Kimya Sanayi Tic. A.S. Ar-Ge Direktorlugu, Ciftlikkoy, Yalova, Turkey $\dagger$ Electronic supplementary information (ESI) available: Mesoscopic morphologies of the fluorinated co-oligomers formed at different concentrations. See DOI: 10.1039/c3cp53079e
}

technologies such as nano-optics, nano-electronics and nanobiotechnology. ${ }^{10,11}$

Low surface energy of the fluorinated polymers is commonly utilized in the area of superhydrophobicity, a subject that became popular when the naturally micro-textured surface of the non-wettable lotus leaves was discovered. ${ }^{12}$ Fundamentally, if a hydrophobic surface is engineered to have mesoscale roughness, or if a mesoscopically rough surface is coated with a low surface energy material, high advancing contact angles are achieved. ${ }^{13-16}$ Previously, we showed that nonhierarchical roughness formed by electrosprayed beads of fluorinated copolymers exhibits non-wettable surfaces despite the existence of polar groups in the structure. ${ }^{17}$ In a subsequent study on the effect of topographical details on wettability, we have synthesized a poly(styrene-co-perfluoroacrylate) random copolymer which yielded an advancing contact angle of $118.5^{\circ}$ on a smooth surface. Among the many parameters which control electrospraying, dielectric constant, viscosity, surface tension, evaporation rate, and solvent quality (relative to the polymer) are the most prominent solvent properties that affect the final coating morphologies. ${ }^{18,19}$ Binary solvent systems are employed to take advantage of different contributions from each solvent, for instance, to provide stable atomization and droplet shape and size control, ${ }^{20}$ control of the spray diameter and reduction of coating roughness, ${ }^{21-23}$ generation of micron and nanometer scale roughness at the same time, ${ }^{24}$ etc. While electrospraying 


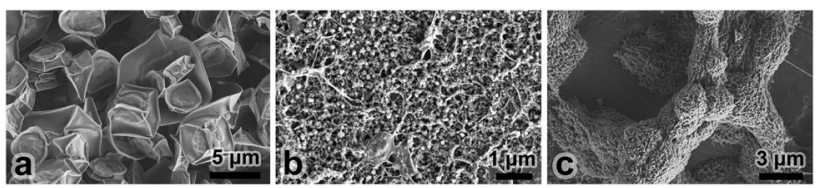

Fig. 1 Typical SEM images of the electrosprayed surfaces of poly(styrene-coperfluoroalkyl ethylacrylate) from solutions of (a) $1 \mathrm{wt} \%$ in THF, (b) $0.15 \mathrm{wt} \%$ in DMF (due to solubility restrictions), and (c) 1 wt \% in THF: DMF (1:1). Applied voltage and solution feed rates were $8 \mathrm{kV}$ and $2 \mu \mathrm{L} \mathrm{min}^{-1}$, respectively, for (a) and (b); and $11.5 \mathrm{kV}$ and $15 \mu \mathrm{L} \mathrm{min}^{-1}$ for (c). Tip-to-ground distance was $10 \mathrm{~cm}$ for all samples. The surface in (c) is perfectly hydrophobic (advancing and receding water contact angles are both $180^{\circ}$ ). Please see ref. 24 for more details on the concepts.

poly(styrene-co-perfluoroacrylate) in a good solvent, tetrahydrofuran (THF), resulted in nanoscopically smooth micron sized beads (Fig. 1a), electrospraying in a poor solvent, dimethylformamide (DMF), yielded nanometer sized particles (Fig. 1b). When the process was carried out in a binary THF-DMF mixture, nanoscopic roughness occurred on micron sized beads (Fig. 1c). Although we predict that formation of these nanoparticles implies a phase separation of the polymer in the poor solvent, ${ }^{24}$ the equilibrium and kinetic processes contributing to the observed behavior are not clear.

In the present study, we investigate the microscopic origin of the solvent dependent morphologies observed for the electrosprayed polymers via numerical simulations and dynamic light scattering (DLS) measurements. Thus, we study the morphological behavior of poly(styrene-co-perfluoroalkyl ethylacrylate) random copolymers in THF, DMF, and THF: DMF (1:1) mixture by dissipative particle dynamics (DPD) simulations. The chain dimensions of the copolymer in these solvents are also studied via molecular dynamics (MD) simulations. Together with the DLS measurements, the results allow interpretation of the observed patterns formed in poor and good solvents as well as their mixtures. In recent experimental studies, ${ }^{21-23}$ solvent types have been used to control roughness in thin films fabricated by an electrospray deposition method. Thus, in this work, we mainly study the equilibrium relationship between polymer and solvent, and we show that this is a prerequisite for controlling morphologies up to a critical applied external force (shearing in this case), whereby we observe that oligomersolvent interactions are maintained. However, we note that electrosprayed surfaces show various and complicated shapes led by not only the equilibrium relationships between polymer and solvent, but also the dynamic process of solvent evaporation. Nevertheless, up to a threshold value, bead size and morphology are not modified by the voltage (see related experimental studies ${ }^{25,26}$ focusing on the relationship between mean particle size and morphology with flow rate and applied voltage). We therefore concentrate mainly on the equilibrium dynamics of polymer-solvent media to disclose the underlying physical mechanisms dominating chain conformations in dual solvent systems, although we discuss solvent evaporation during electrospraying in the subsection Formation of dual scale roughness via competing solvents.

\section{Methods and materials}

\section{Materials and the electrospraying process}

The poly(styrene-co-perfluoroalkyl ethylacrylate) random copolymer, having $13 \mathrm{~mol} \%$ fluorinated monomer, has a number average molecular weight and polydispersity index of $105600 \mathrm{~g} \mathrm{~mol}^{-1}$ and 1.8 , respectively. Synthesis of the copolymer is described in detail in the supporting information of ref. 24. Tetrahydrofuran (THF, Merck) and $N, N$-dimethylformamide (DMF, Merck) were used as received. Electrospraying experiments were performed using a Gamma High Voltage ES30 power supply for electrostatic deposition and a New Era NE-1000 syringe pump to control the solution feed rates. The setup also comprised a grounded aluminum plate having a $10 \mathrm{~cm} \times 10 \mathrm{~cm}$ collecting screen. In the set-up, the syringe pump was placed such that the axis of the cylindrical syringe on it lies parallel to the ground and its charged tip is normal to the plane of the collecting screen standing at a distance of $10 \mathrm{~cm}$.

\section{Particle size measurements}

We perform DLS analysis to (i) obtain information on relative dimensions of single polymer chains, and (ii) assess the propensity of agglomerate formation in a given solvent environment. To avoid multiple scattering phenomena in DLS measurements and to obtain an accurate profile of single chain conformations, solutions in a dilute regime were employed. This choice of regime is in contrast to electrospraying conditions whereby the semi-dilute unentangled concentration regime must be employed to achieve polymeric beads. ${ }^{27}$

Particle size analyses of the samples were performed using DLS technique with a Malvern Instruments Zetasizer Nano-ZS. DLS measures the dynamic fluctuations of scattered light intensity from the Brownian motion of the particles in liquid media and performs a velocity distribution analysis, which may be correlated to a hydrodynamic radius via the Stokes-Einstein equation. ${ }^{28}$ In each analysis, $3.0 \mathrm{mg}$ of the polymer was transferred into $15 \mathrm{~g}$ of the corresponding liquid and stirred rigorously using a magnetic stirrer for $30 \mathrm{~min} .1 .0 \mathrm{~mL}$ of the dispersion was gently transferred into a quartz cuvette and a total of 90 measurements were averaged from three different batches that belong to the same dispersion. Note that we have also conducted DLS measurements after heating the solutions, and even by ultrasonicating at high temperatures prior to analysis to overcome the solubility limit in DMF. However, we did not observe any difference in particle size distributions, confirming the stability of the equilibrium states in the poor solvent.

\section{MD simulations of solvated single polymeric chains}

MD simulations provide an atomistic level picture of structure and dynamics. As is the case for polymer-solvent interactions, MD simulations allow prediction of material properties that rely on atomistic detail as well as interpreting their observed behavior. In this study, we use MD simulations to predict the dimensions of single chains. The molecular simulation software package Materials Studio ${ }^{\circledR} 6.0^{29}$ was used to construct the 
Table 1 Root mean square end to end distance $\left\langle R_{\text {end }}{ }^{2}\right\rangle^{1 / 2}$ and concentration (wt\%, in brackets) for the simulated single chains of fluorinated copolymers in different solvents $(P=1 \mathrm{~atm}, T=300 \mathrm{~K})$; standard error on the mean is also indicated

\begin{tabular}{|c|c|c|c|c|}
\hline & \multicolumn{4}{|c|}{ 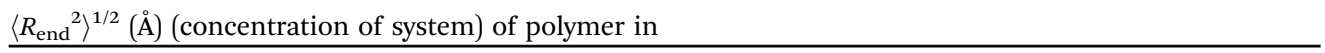 } \\
\hline Number of monomers, $N$ & $\begin{array}{l}10\left(\mathrm{~N}_{10}\right) \\
20\left(\mathrm{~N}_{20}\right) \\
40\left(\mathrm{~N}_{40}\right) \\
80\left(\mathrm{~N}_{80}\right) \\
100\left(\mathrm{~N}_{100}\right) \\
200\left(\mathrm{~N}_{200}\right)\end{array}$ & $\begin{aligned} 20.28 & \pm 0.45(0.04) \\
29.5 & \pm 1.1(0.06) \\
47.46 & \pm 0.53(0.12) \\
65.55 & \pm 0.61(0.22) \\
78.84 & \pm 0.04(0.27) \\
114.34 & \pm 0.48(0.53)\end{aligned}$ & $\begin{aligned} 21.12 & \pm 0.32(0.04) \\
30.48 & \pm 0.81(0.07) \\
49.56 & \pm 0.99(0.12) \\
65.88 & \pm 0.80(0.22) \\
76.5 & \pm 0.64(0.27) \\
114.72 & \pm 1.07(0.53)\end{aligned}$ & $\begin{aligned} 14.4 & \pm 0.55(0.04) \\
32.4 & \pm 0.41(0.07) \\
47.16 & \pm 0.73(0.12) \\
68.73 & \pm 0.45(0.22) \\
75.41 & \pm 1.02(0.27) \\
111.51 & \pm 0.54(0.53)\end{aligned}$ \\
\hline
\end{tabular}

Table 2 Flory characteristic ratio $\left(C_{n}\right)$ for the simulated single chains of fluorinated copolymers in different solvents $(P=1$ atm, $T=300 \mathrm{~K})$; standard error on the mean is also indicated. Average cell dimensions ( $\AA$ ) of the equilibrated systems are displayed in brackets

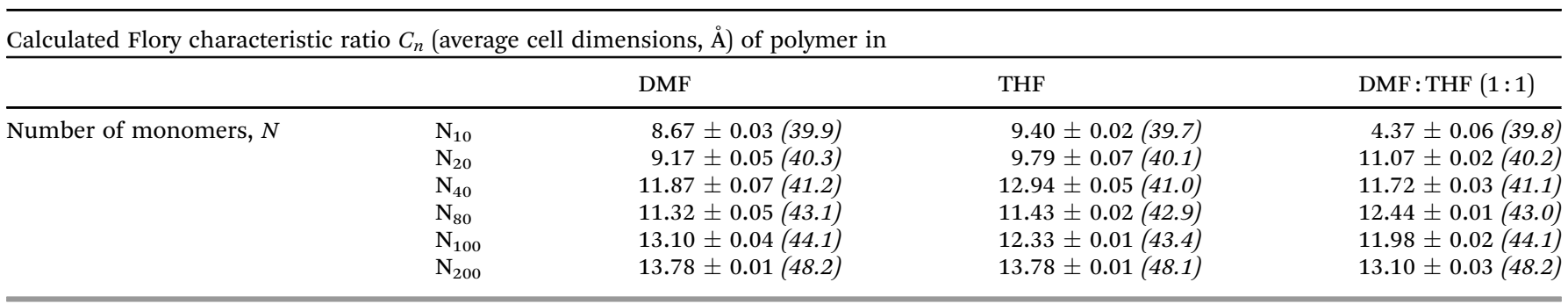

initial molecular structures and simulations and for post-processing the collected trajectories. COMPASS (Condensed-phase Optimized Molecular Potentials for Atomistic Simulation Studies) force field $^{30}$ used in this study has been shown to be very effective in defining the properties of synthetic polymers. Simulation boxes constructed using the Amorphous Cell module contained 500 molecules of solvent and a single polymer chain at a target density of $1.0 \mathrm{~g} \mathrm{cc}^{-1}$. Well-relaxed atomistic configurations of the model poly(styrene-co-perfluoroalkyl ethylacrylate) in three different solvent systems, THF, DMF, and THF : DMF (1:1), with the number of monomers in the range $N=10-200$, were subjected to detailed molecular dynamics simulations for simulation times of up to $5 \mathrm{~ns}$. In addition, polymers of 80 , 100 , and 200 repeat units were initially subjected to $20 \mathrm{~ns}$ vacuum simulations to relax the polymer structure prior to cell construction. MD simulations of the solvated systems were carried out in the isothermal-isobaric (NPT) statistical ensemble, at $P=1 \mathrm{~atm}$ and $T=300 \mathrm{~K}$. To maintain temperature and pressure at their prescribed values, the Andersen-Berendsen thermostatbarostat was used. ${ }^{31,32}$ Results for root mean square end to end distance $\left\langle R_{\mathrm{end}}{ }^{2}\right\rangle^{1 / 2}$ and the average cell dimensions are presented in Table 1 . The concentration of the equilibrated systems and the Flory characteristic ratio $\left(C_{n}\right)$ are listed in Table 2.

\section{DPD parameterization}

DPD simulations are performed on a collection of particles, each made up of a suitably selected set of atoms (see below). The force acting on a given bead at each time step is the sum of soft repulsive forces acting along the line connecting the bead to its neighbors, a dissipative term and a random force term. In addition, the beads that are located consecutively along the contour of a chain are connected by a harmonic force.

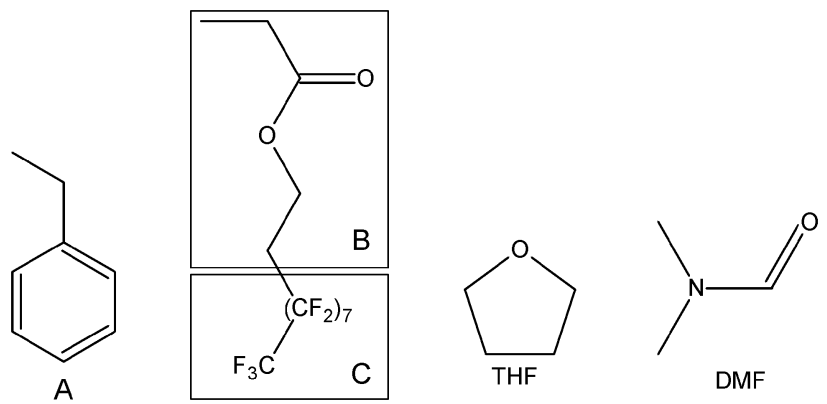

Fig. 2 Partitioning of the beads for coarse-grained simulations. THF and DMF molecules are whole units.

The phase behavior of styrene-co-fluorinated chains in THF was previously studied by DPD simulations in our group. ${ }^{33}$ We therefore provide a brief overview of the parameterization methodology used and refer the reader to that manuscript for details. In a DPD simulation, the chains need to be partitioned into beads made up of chemically distinct units that also have similar sizes. The units we use in the current study are displayed in Fig. 2, whereby the $\mathrm{A}, \mathrm{B}$, and $\mathrm{C}$ units represent the styrene, ethyl acrylate and perfluoroalkyl segments, respectively. Each solvent molecule is treated as a separate unit, THF or DMF.

As in our previous work and following the established protocol, Hildebrand solubility parameters $(\delta)^{34}$ were calculated from successive 1 ps equilibration steps and 100 ps MD simulations on simulation boxes that contain 10 beads of the same type with a density of $1.0 \mathrm{~g} \mathrm{cc}^{-1}$ at $298 \mathrm{~K}$, using the Amorphous Cell and Forcite modules of Materials Studio ${ }^{\circledR} \cdot{ }^{29}$ For all non-bonded interactions, a cut-off radius, $r_{\mathrm{c}}$, of $8.5 \AA$ and periodic boundary conditions were applied in the canonical ensemble (NVT). The molar volume of the beads, $V_{\mathrm{m}}$, was 
Table 3 Properties of beads as defined in Fig. 2. Solubility parameters, $\delta$, molar volume $V_{m}$, Flory-Huggins interaction parameters, $\chi_{i j}$ (upper diagonal, shown in bold) and DPD interaction parameters, $a_{i j}$ (lower diagonal)

\begin{tabular}{lccccc}
\hline & $\mathrm{A}$ & $\mathrm{B}$ & $\mathrm{C}$ & $\mathrm{THF}$ & $\mathrm{DMF}$ \\
\hline$\delta\left(\mathrm{cal} \mathrm{cm}^{-3}\right)^{1 / 2}$ & 7.99 & 9.33 & 3.92 & 9.31 & 12.29 \\
$V_{\mathrm{m}}\left(\mathrm{cm}^{3} \mathrm{~mol}^{-1}\right)$ & 115.3 & 109.6 & 166.4 & 79.7 & 83.0 \\
$\mathrm{~A}$ & $\mathbf{0 . 0 0}$ & $\mathbf{0 . 2 9}$ & $\mathbf{4 . 1 0}$ & $\mathbf{0 . 2 1}$ & $\mathbf{3 . 0 9}$ \\
$\mathrm{B}$ & 25.00 & & & & \\
$\mathrm{C}$ & 25.95 & 25.00 & 7.24 & $\mathbf{0 . 0 0}$ & $\mathbf{1 . 4 2}$ \\
& & & $\mathbf{0 . 0 0}$ & $\mathbf{3 . 4 5}$ & $\mathbf{1 4 . 7 5}$ \\
THF & 38.42 & 48.69 & 25.00 & & \\
& & & & $\mathbf{0 . 0 0}$ & $\mathbf{1 . 2 2}$ \\
DMF & 25.67 & 25.00 & 36.27 & 25.00 & $\mathbf{0 . 0 0}$ \\
& 35.13 & 29.66 & 73.25 & 29.00 & 25.00 \\
\hline
\end{tabular}

calculated using the ACDLabs/Chemsketch 5.0. ${ }^{35}$ Solubility parameters were determined according to,

$$
\delta=\left(\frac{\Delta E_{\mathrm{v}}}{V_{\mathrm{m}}}\right)^{1 / 2}=(\mathrm{CED})^{1 / 2}
$$

where $\Delta E_{\mathrm{v}}$ and CED correspond to molar energy of vaporization and cohesive energy density, respectively. The Flory-Huggins interaction parameter, $\chi$, of the beads was then calculated from,

$$
\chi=\frac{V_{\mathrm{m}}\left(\delta_{i}-\delta_{j}\right)^{2}}{k_{\mathrm{B}} T}
$$

where $V_{\mathrm{m}}$ is the average molar volume of the beads $i$ and $j$. The calculated values of $\delta$ and $\chi$ are displayed in Table 3 . The relatively close $\delta$ values between the bead-pairs A, B, C, and THF indicate that solubility will be promoted in THF. On the other hand, the distinct values for DMF interactions, particularly for the perfluoroalkyl segment C, imply non-preferred solubility in DMF. These results are consistent with the experimental solubility values for the polymer, which are greater than $30 \mathrm{wt} \%$ in THF and $0.15 \mathrm{wt} \%$ in DMF.

$\chi$ is related to the thermodynamics of mixing, and the DPD interaction parameter, $a_{i j}$, obtained from $\chi$ is a measure of repulsion between the beads. The latter is calculated using the linear relationship put forth by Groot and Warren ${ }^{36}$ as $a_{i i}=$ $25 k_{\mathrm{B}} T$ and $a_{i j} \approx a_{i i}+3.27 \chi_{i j}$ for a box density of 3 DPD units (Table 2). We note that this treatment assumes equal repulsive interactions between like beads, which is strictly true only when the molar volumes of the two components are equal. ${ }^{37}$ In this work, we are interested in the qualitative behavior of the chains in the investigated solvents, and we extend on our previous work for which we have already shown that the values for beads A, B, C, and THF lead to physically observed phases. ${ }^{33}$ We therefore continue with that approach.

\section{DPD simulations}

For DPD simulations, an oligomeric chain of 100 monomers was constructed by using the beads shown in Fig. 2. Cubic boxes having a $10 \times 10 \times 10 u^{3}$ volume are constructed with a density of 3 DPD units where $u$ is the cut-off radius. A harmonic spring constant of 4.0 was chosen for beads located consecutively along the chain. Temperature and bead masses were taken as unity. The total number of beads was set to 3000 . The systems were equilibrated for 20000 DPD steps, followed by 100000 DPD steps for data collection and analysis.

We study the morphologies of the multi chain-solvent systems at a series of concentrations $(5,10,20,30,40,50$, and $70 \%$ polymer by volume). In this manner, we observe the concentration dependent phase behavior of the polymer in the solvents. We note that the chains are random copolymers of $90 \mathrm{~A}$ and 9 (B-C) beads. This is in contrast to our previous work where we studied block copolymers of the same composition. ${ }^{33}$ For selected compositions, we also study the phase behavior of the systems under shear to test the extent of stability of the phases observed.

Inasmuch as electrospraying involves shearing of chains in the solvent environment, the stability of the morphologies is also studied by applying steady shear to the simulation box using sliding brick boundary conditions ${ }^{38}$ as implemented in the Materials Studio ${ }^{\mathbb{R}}$ package program.

\section{Results and discussion}

\section{DLS analyses reveal the nonlinear effect of a binary solvent system on chain dimensions}

DLS is a powerful tool providing insight into the hydrodynamics of real chains in the solvent. Hydrodynamic radii of the chains are calculated from diffusion coefficients using the Stokes-Einstein equation, assuming that the particles of the samples are hard spheres. However, the polymer chains are expanded coils in good solvents, thus the hard sphere assumption is not sufficient to explain the dissolved polymer geometry. The hydrodynamic radius, $R_{\mathrm{H}}$, is proportional to the root mean square end-to-end distance $\left\langle R_{\text {end }}\right\rangle^{1 / 2}$. Single chain dynamics and chain dimensions in different solvents obtained from MD simulations will be discussed in the next subsection.

DLS measurements of the copolymer in the good solvent revealed a single peak at $13.0 \mathrm{~nm}$. This small Gaussian peak, or the absence of multiple peaks, demonstrates that the copolymer dissolves homogenously in THF. The single phase dissolution of copolymer in THF also leads to smooth surfaced electrosprayed micron size particles as seen in Fig. 1a. Conversely, DLS measurements in a different medium, DMF, lead the copolymer to exhibit a larger peak at around $265 \mathrm{~nm}$, implying aggregates of polymer chains. Since scattering intensity has strong dependence on particle size (proportional to the sixth power of diameter), ${ }^{39}$ we filtered the solution with a filter having $200 \mathrm{~nm}$ diameter pore size and repeated the measurement to detect peaks corresponding to relatively small particle sizes. We then observed the peak corresponding to single chains with an average hydrodynamic radius of $12.2 \mathrm{~nm}$, because of the lowered scattering intensity from the aggregates whose number is reduced after filtration. The smaller hydrodynamic radius of the chains observed in DMF indicates that they are more compact in DMF than in THF. In the binary THF: DMF (1:1) mixture, however, the chain dimension is lower than it is in either of the corresponding pure solvents.

We propose that in a binary solvent system, the THF-DMF interactions must be influencing the chain conformation. 
Thus, additional repulsive forces between DMF-THF solvent molecules lead to encapsulation of the chain, as illustrated in Fig. 4. In the next section, we report MD simulation results (i) to interpret the chain dimensions in different media, while confirming that the first peak in the DLS results indeed corresponds to single chains, and (ii) to determine the distribution of the solvent molecules around the chain by calculating radial distribution functions (RDFs).

\section{Single chain dynamics observed by MD simulations corroborate DLS analyses}

In the previous section, we emphasized that the hydrodynamic radius $\left(R_{\mathrm{H}}\right)$ is proportional to the root-mean-square end-to-end distance $\left\langle R_{\text {end }}^{2}\right\rangle^{1 / 2}$. These measures of chain size carry information on the local chain geometry, which depends on valence and dihedral angle distributions as well as local interactions. They also correlate with experimentally measurable global dimensions, which are a combination of packing effects and local intermolecular interactions. Accordingly, $\left\langle R_{\text {end }}{ }^{2}\right\rangle^{1 / 2}$ values of chains having different numbers of monomers in THF, DMF, and THF:DMF (1:1) were calculated and are listed in Table 1 . By extrapolating to infinite chain length, we estimate Flory's characteristic ratio $C_{\infty}$ in THF, DMF, and binary solvent systems as:

$$
\left\langle R_{\text {end }}{ }^{2}\right\rangle=C_{\infty} n l^{2}
$$

where $n$ is the number of units along the backbone $(n=2 N$ in this case) and $l$ is the average skeletal bond length, which is approximately $1.54 \AA$ in our system. Thus, the $C_{\infty}$ values in THF, DMF, and THF : DMF $(1: 1)$ at $300 \mathrm{~K}$ are estimated as 14 , 13 and 12, respectively. The order of calculated values is in agreement with the first peaks of the DLS curves (Fig. 3).

We rationalize this outcome by considering the net excluded volume of the chain in each case as predicted by examining the interaction parameter, $\chi$, (Table 3 ). All units of the chain have a smaller net repulsion towards THF than DMF. The $\chi$ parameters are 0.21 vs. 3.09 for A bead-THF and A bead-DMF interactions, respectively. Similarly, they are 3.45 (THF) vs. 14.75 (DMF) for the C units. B units are insensitive to THF while their $\chi$ parameter in DMF is 1.42. Meanwhile, the $\chi$ parameter for $\mathrm{A}-\mathrm{C}$ interactions is 4.1 , which is larger than both of the A-THF and C-THF interactions. Thus, the net result in THF is expansion, leading to a positive excluded volume. In DMF, however, the large $\chi$ parameter of C-DMF units overcomes the intrinsic repulsions between chain units leading to a net negative excluded volume.

To provide insight into why the chain has smaller dimensions in the THF : DMF $(1: 1)$ mixture than in either of the pure solvents, we have studied the RDF of the equilibrated trajectories. All atoms in a given unit are chosen as centers in the RDF calculations. The results obtained from the $\mathrm{N}_{40}$-binary solvent and single solvent systems are displayed as a representative in Fig. 5. We find that in the single solvent systems, the distribution of solvent molecules around the chain is similar in DMF and THF. The first coordination shell of oligomer-solvent interactions ends at $c a .6 .8 \AA$ in both cases, with DMF displaying sharper distributions than THF. However, the solvent-solvent interactions in DMF are much stronger than those in THF. In contrast, binary solvent systems are modified such that the DMF-copolymer interactions at the interface are suppressed and that the probability of finding THF near the chains is substantially higher. The presence of a well-defined first peak is the signature of a net attraction between the chain and the THF molecules, which is now absent for DMF.

Because of the tendency of polymeric units to prefer THF molecules over DMF, there will be an uneven distribution of THF and DMF molecules elsewhere. This is in fact observed in the RDFs of binary solvent systems (Fig. 5b), where, in the presence of THF, DMF molecules tend to cluster more with the



Fig. 3 DLS analysis of $0.02 \mathrm{wt} \%$ polymer in THF, THF : DMF (1:1), DMF and DMF after passing the solution through a filter having $200 \mathrm{~nm}$ diameter pores. Peaks

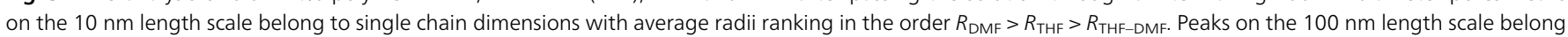
to agglomerates. 
A



Fluorinated Copolymer in THF
B

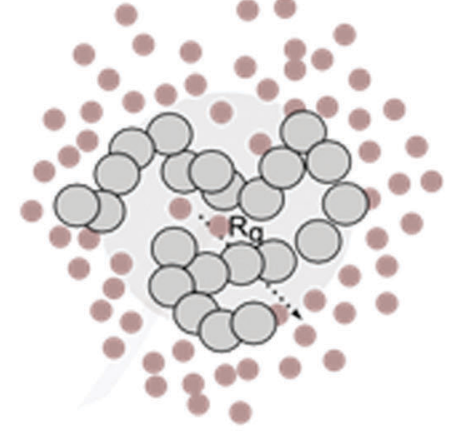

Fluorinated Copolymer in DMF

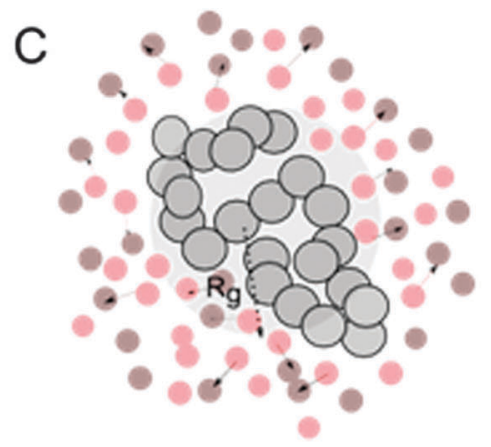

Fluorinated Copolymer in THF-DMF

Net repulsion between

DMF and THF solvent molecules

Fig. 4 Illustrated model for the effect of solvent on chain conformation.

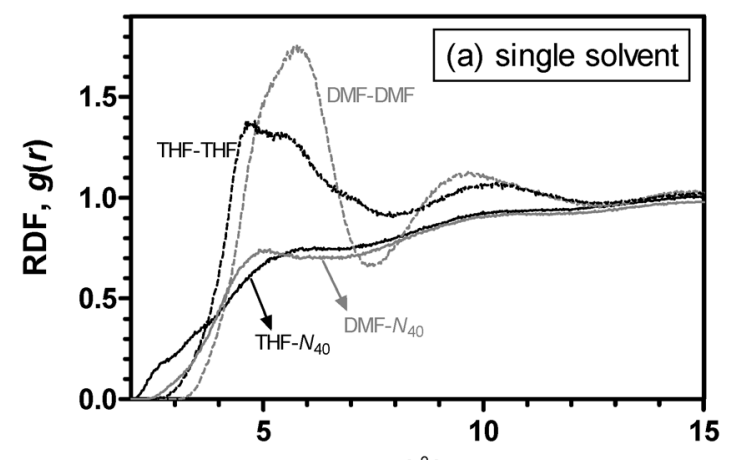

$r(\AA)$

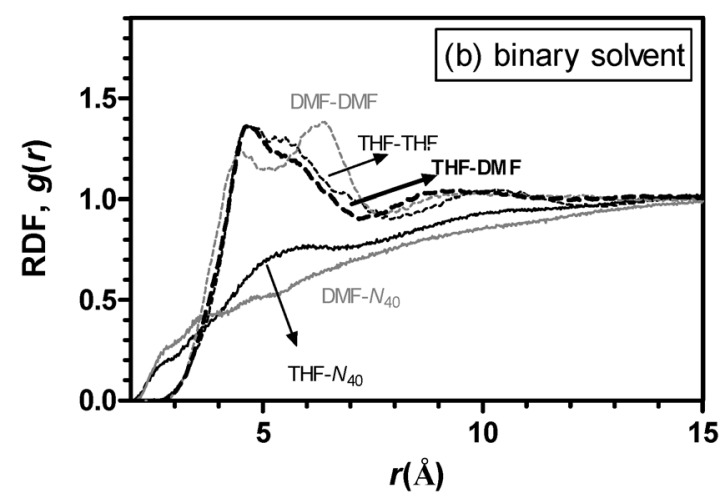

Fig. 5 Radial distribution function $g(R)$ of an $\mathrm{N}_{40}$ polymer chain in the binary THF : DMF $(1: 1)$ mixture. Similar results are obtained for $\mathrm{N}_{10}-\mathrm{N}_{80}$ systems.

other species than its own kind. Thus, we observe that the THF-THF distributions are not altered in the binary solvent while DMF-DMF distributions shift significantly. Due to slight repulsions between THF and DMF molecules $(\chi$ parameter of THF-DMF is 1.22), the incorporation of THF molecules into DMF brings additional repulsive forces into the system. Overall, the attractive force between THF and DMF overcomes the overall attractive forces between DMF and polymer, resulting in the contraction of the chain (see Fig. 4 for the scheme depicting the distribution of solvent molecules around the chain).

MD and DLS analyses unveil single chain dynamics in the different solvents. The small variations observed in chain dimensions and solvent ordering around a single chain lead to large morphological differences in many chain systems of the same polymeric systems, which we investigate next.

\section{Mesoscopic morphologies monitored by DPD simulations rationalize experimental observations}

To interpret the aggregation behavior observed in electrosprayed samples (Fig. 1) and DLS analyses (Fig. 3), we employed DPD simulations to predict the many-chain morphologies of the polymers in the solvents at equilibrium. The morphologies of the three systems at $10 \%$ concentration at equilibrium and under various shear rates are displayed in Fig. 6. In addition, the morphologies obtained at all concentrations are given in ESI $\uparrow$ (Fig. S1). Note that we do not expect a one-to-one correspondence between the DPD morphologies obtained and the electrosprayed surfaces observed. The mesoscopic morphologies merely depict how chains aggregate in the presence of the different solvent environments.

The DPD simulations predict the equilibrium morphologies of the block copolymers as homogenous mixtures in THF (Fig. 6a). This result is in agreement with the single chain behavior in the respective solvents. Although copolymers have a $13 \mathrm{~mol} \%$ fluorinated group (segment C), similar DPD parameters for monomermonomer and monomer-solvent interactions promote miscibility and prevent micro-phase separation in THF. This finding is in accord with DLS results which emphasize that the copolymer forms a homogenous solution with THF with a single Gaussian peak centered on the average single chain size (Fig. 3).

Conversely, due to strongly repulsive interactions between A-DMF and C-DMF beads, phase separation occurs even at low concentrations of the polymer in DMF (Fig. 6b). The order of the appearing phases as the concentration is increased is 
a)THF



b)



$0.01^{*}$
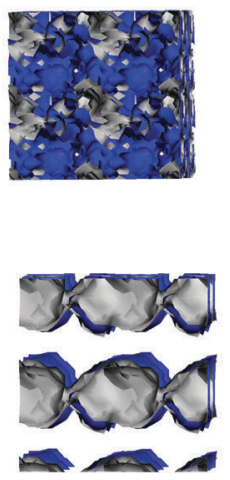

$0.05^{*}$
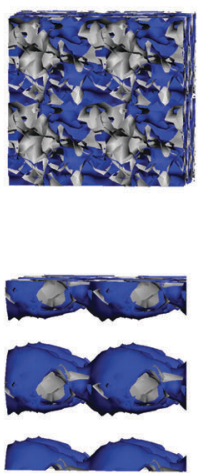

$0.1^{*}$
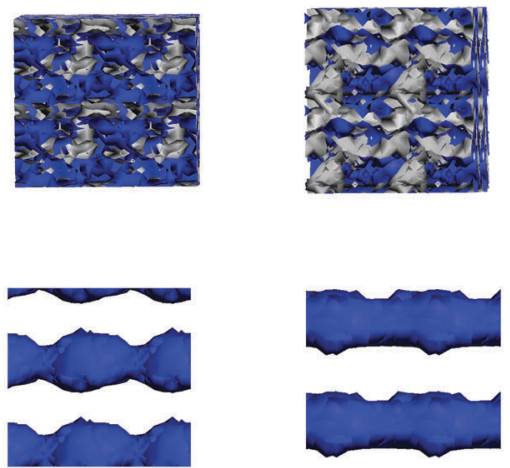

$0.2^{*}$

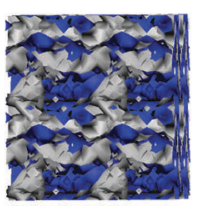

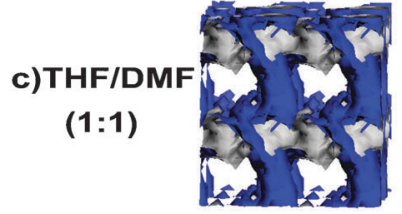
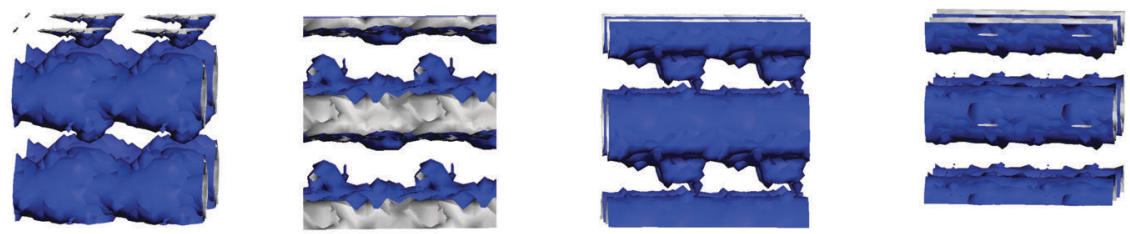

Fig. 6 Mesoscopic morphologies of the fluorinated co-oligomers formed at equilibrium and under various shear rates. Representative snapshots are presented for $10 \%$ concentrations in (a) THF, (b) DMF, and (c) THF : DMF (1:1). Periodic images of the simulation boxes are reproduced in $2 \times 2$ format for better visualization of the phases formed. The shapes are represented as isosurfaces around all the oligomeric beads. In (b), phases are always clearly separated; conversely, in (c) a dynamic bridging between the microphases is established except at the largest shear rate.

typical of those observed for surfactants (sphere $\rightarrow$ cylinder $\rightarrow$ lamella $\rightarrow$ reverse micelles) ${ }^{40}$ (see Fig. S1, ESI $\dagger$ for DMF). The stability of the formed phases is tested by applying shear, since resistance to mixing under shear occurs in stable micelles. ${ }^{41}$ The spherical phase formed at $10 \%$ concentration deforms into distorted spheres at low shear rates, undulated structures at moderate shear rates, and cylinders at high shear rates (Fig. 6); the clear phase separation between the chains and the solvent is maintained at all shear rates. No notable change is observed in the THF system and the homogeneity is conserved.

A detailed investigation of the structures formed during the DMF simulations reveals that solvent molecules cannot break up polymer aggregates or penetrate into the structure even under shear. Thus, the second peak observed in the DLS analysis at $\sim 265 \mathrm{~nm}$ (Fig. 3) is attributed to the agglomerated polymer chains due to lack of swelling in DMF. This is the case even at very low polymer concentrations, obtained by filtering the solution, implying that the critical micelle concentration (CMC) for this system is very low. CMC of the DMF-polymer system is quite low compared to the THF-polymer system.

The shifting of the peak locations upon filtering relies on the large change that is expected to occur in the distribution of molecules found in an aggregate as a function of concentration near the CMC. ${ }^{42}$ Therein, it has been shown that a large peak at the aggregate size appears above the CMC. Conversely, below the CMC, a large peak belonging to monomer size is accompanied by a small one at the micelle sizes (see Fig. 16.6 in ref. 30). Consequently, filtering larger agglomerates changes polymer concentration in DLS solution and causes the distribution to converge to the profile observed below the CMC. Thus, although all the particles with average diameters larger than $200 \mathrm{~nm}$ are expected to be trapped in the filter, new aggregates may form with a similar aggregation number at a new equilibrium at lower surfactant concentration. Our experimental observations point out that polymer concentrations exceeding $0.15 \mathrm{wt} \%$ lead to precipitation in DMF; this is the concentration we present in Fig. $1 \mathrm{~b}$.

DPD simulations of the copolymers in the binary solvent mixture yield a different morphology. When isosurfaces around the A, B, and C beads are generated (Fig. 6c), structural features which are far from those that would result in complete mixing, are observed. As displayed by the sample snapshots in Fig. 7, the chains are in dynamic equilibrium between completely solvated single chains and clusters of several chains. To interpret the structural details of the morphologies, we have calculated the RDFs of the fluorinated $\mathrm{C}$ beads (Fig. 8a). Also displayed in this figure are snapshots of chain morphologies. Note that these morphologies are obtained even in a two-chain system represented by $5 \%$ DPD simulations (Fig. S2, ESI $\dagger$ ).

For systems solvated in THF, there is a lack of structure between $\mathrm{C}$ beads residing on different chains. In DMF, the solvophobic $\mathrm{C}$ beads tend to cluster together, yielding a strong peak at around 5 DPD units. In the binary solvent, we find a tendency of the $\mathrm{C}$ beads clustering together, although it is not as strong as it is in pure DMF. Thus, the average structures in the binary solvent have the form of interconnected swollen 
THF/DMF (1:1)

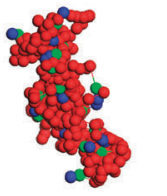

20000 DPD steps

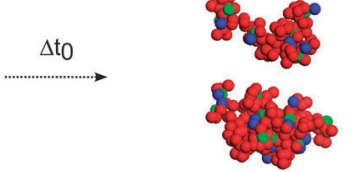

40000 DPD steps



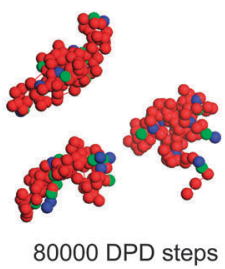

$\Delta \mathrm{t}_{2}$

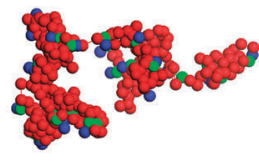

100000 DPD steps

Fig. 7 Snapshots of chains in THF : DMF (1:1) mixture revealing the dynamical equilibrium between single chains (left) and clusters of various sizes.
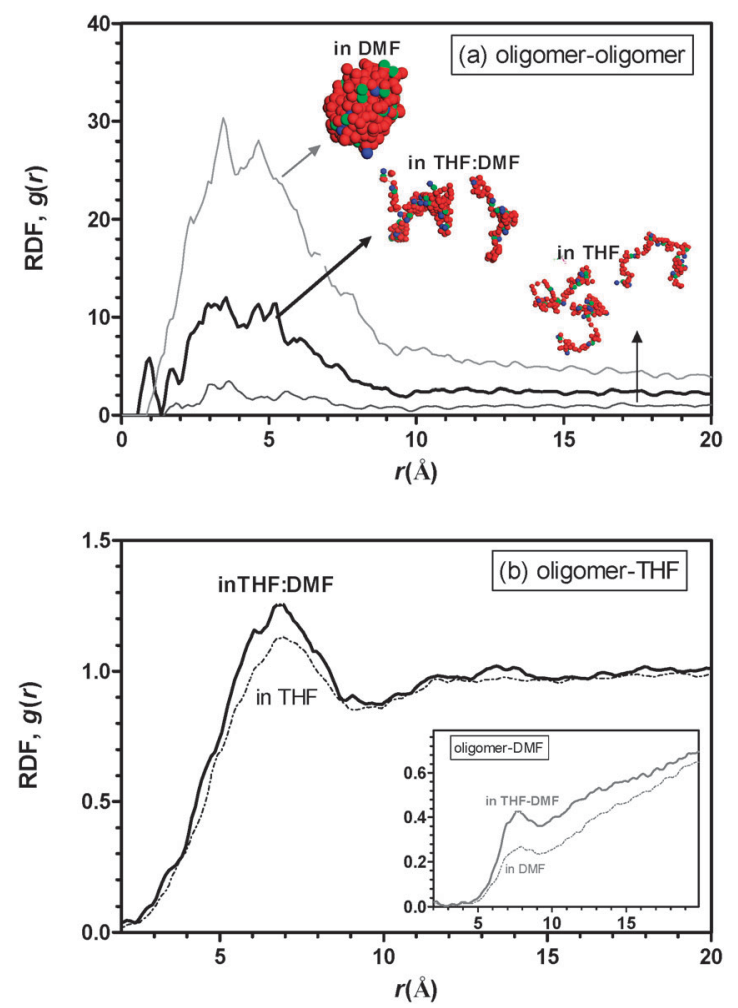

Fig. 8 RDFs of fluorinated $C$ beads compared in different solvent environments: (a) distribution of $C$ beads residing on other chains around the $C$ beads; (b) distribution of THF beads in binary and single solvent systems around the $C$ beads; those of DMF beads which cannot get into close contact with the chains are displayed as inset.

chain clusters, whereby fluorinated groups tend to stay close to each other while the solvent penetrates into them. We note that the regions between chains are THF rich, while the outer parts are isolated by DMF molecules. This observation is corroborated by the RDFs of the solvent beads around the fluorinated beads studied in the single versus binary solvents (Fig. 8b). Abundance of THF beads around the fluorinated segments is expected in the completely miscible THF-copolymer mixture, and this is further enhanced in the binary solvents. Perhaps more important, the fluorinated segments, which are almost completely shielded from the DMF molecules in the DMFcopolymer mixture, tend to stay away from the DMF units even in their swollen states in the binary mixture (see inset to Fig. 8b). Although there is substantial structure in the polymeric units dissolved in the binary solvent, the high degree of solvent penetration into the polymeric clusters, as well as the dynamical equilibrium observed between single chains and multiple chain clusters, lead to the lack of peaks belonging to large agglomerates in the DLS analyses (Fig. 3). Finally, the dynamical equilibrium of chains between completely solvated single chains and clusters of several chains is observed up to the largest shear rate value of 0.2 , at which point complete phase separation occurs (Fig. 6c).

\section{Formation of dual scale roughness via competing solvents}

Electrospinning involves drawing an electrically charged jet of polymer solution towards a grounded collector and formation of nano or microfibers upon elongation and thinning of this jet prior to solidification. ${ }^{17,43-45}$ If the solution is at a semi-dilute unentangled concentration regime, this jet disintegrates into micron sized droplets. The formation of super hydrophobic surfaces is possible once these droplets of a hydrophobic polymer dry out on a film.

To describe drying phenomena in films, a mathematical model that involves the solution of the diffusion equation with an exponential variation in the diffusion coefficient, $D$, and a surface boundary resistance (quantified by surface mass transfer coefficient, $h$ ) has been used. ${ }^{46}$ Note that drying phenomena involve the concentration dependent diffusion of solvents since their diffusion coefficient is expected to be different near the polymeric chains than near the air interface. Furthermore, convective effects, such as blowing air past the surface, substantially reduce the boundary layer resistance.

Drying - loss of solvent - from a polymeric film has contributions from (i) the boundary layer resistance to solvent transport into vapor, and (ii) the internal diffusion resistance to solvent loss, as illustrated in Fig. 9. In the initial stage of drying, the droplets may be considered uniform in concentration since the diffusivities are high, allowing free movement of solvent within the polymer. This stage is defined mostly by the evaporation rate of solvents. Later on, the main resistance to solvent loss occurs at the air interface. As a result, at short times, the boundary layer resistance controls the drying process with a cross over at moderate times into a diffusion controlled regime. Thus, the two material properties that characterize drying rates and dynamics are $D$ and $h$ of the solvent molecules.

In light of these factors, drying both in electrospinning and electrospraying experiments has three phases: (a) for the short duration the feed is in the air, boundary layer resistance is very low ( $h$ is very high) and drying occurs freely; (b) when the jet of polymer solution lands on the collector, $h$ is substantially reduced. If there is solvent remaining in the sample, it will be 


\section{electric field}

\section{$1^{\text {st }}$ drying stage}

boundary layer resistance to solvent transport into vapor (surface mass transfer coefficient, $h$ )

$$
\mathrm{h}_{\mathrm{THF}}>\mathrm{h}_{\mathrm{DMF}}
$$

A) THF

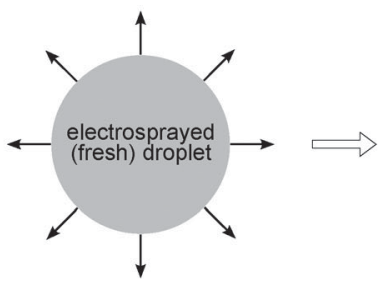

B) DMF

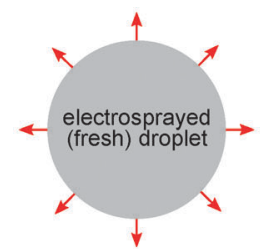

C) $\mathrm{THF}+\mathrm{DMF}$

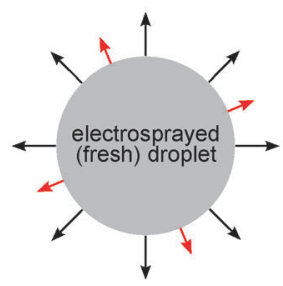

Evaporation of THF molecules

Evaporation of DMF molecules
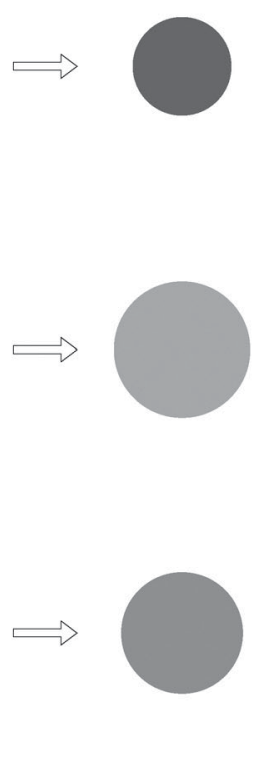

1

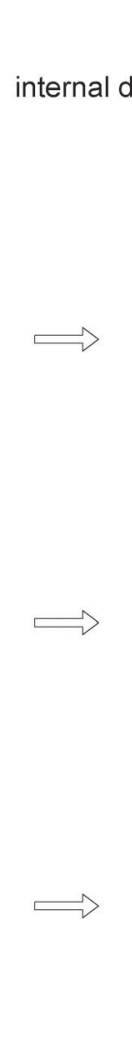

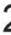

$2^{\text {nd }}$ drying stage

(diffusion coefficient, D)
(iffusion resiance to sol

$\mathrm{D}_{\mathrm{THF}}>\mathrm{D}_{\mathrm{DMF}}$
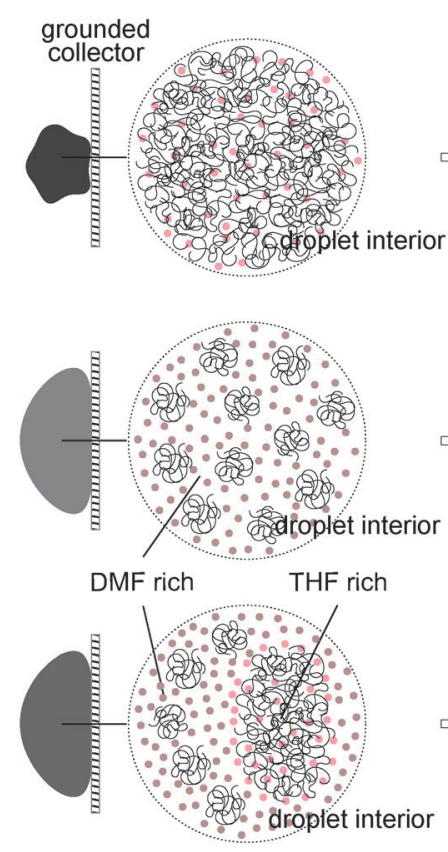

- THF molecules

DMF molecules

Polymer chains

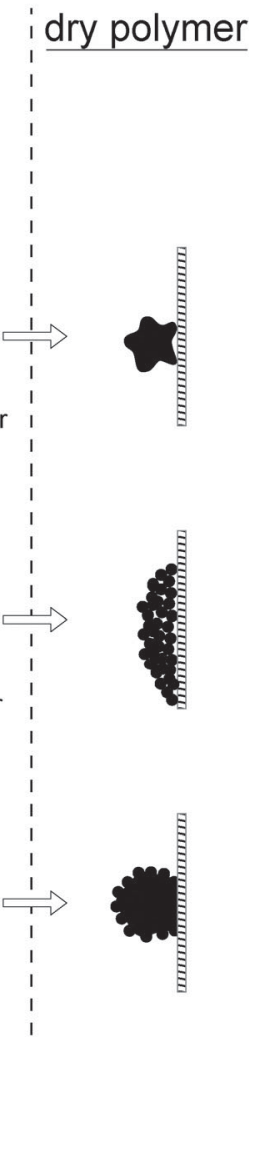

Fig. 9 Illustration of the effect of the drying process on the formation of dual-scale roughness.

subject to boundary resistance controlled drying; (c) the time scale of diffusion control is reached on the sample on the collector. Phase (b) may or may not occur depending on how much $h$ is modified by the feed rate and applied voltage in phase (a) and the rate of diffusion relative to the result of these effects. Yoshida and Miyashita ${ }^{47}$ indicated that the lowerboiling solvent is always removed preferentially and its selectivity is almost constant irrespective of the initial composition and the thickness of the layer. Solvent characteristics should be designated to control the drying process as well as the surface morphology of films.

DLS, MD, and DPD analyses all indicate that the copolymer dissolves in THF (Fig. 3 and 6a). Furthermore, THF is very volatile (boiling point is $66^{\circ} \mathrm{C}$, relative evaporation rate (butyl acetate $=1)$ is 6.3$)^{18}$ and has low viscosity $\left(0.46 \times 10^{-3} \mathrm{~Pa} \mathrm{~s}\right)$, thus yielding high $h . D$ of THF is also high in polymer media. (Note that we have estimated diffusivity of the solvents in the polymer from single chain MD simulations and found that $D_{\mathrm{THF}}$ is slightly higher than $D_{\mathrm{DMF}}$, which are $1.54 \times 10^{-9}$ and $1.44 \times 10^{-9} \mathrm{~m}^{2} \mathrm{~s}^{-1}$, respectively. On the other hand, selfdiffusivity of THF, $D_{\text {self-THF }}=3 \times 10^{-9} \mathrm{~m}^{2} \mathrm{~s}^{-1}$, is much higher than $D_{\text {self-DMF }}=7 \times 10^{-10} \mathrm{~m}^{2} \mathrm{~s}^{-1}$.) Thus, there is competition between boundary resistance and diffusion resistance to drying. At high voltages, more of the drying is expected to take place in stage (a), not allowing the chains enough time to coalesce. At lower voltages, stages (b) and (c) will also be effective with enough solvent remaining to allow larger particles to be observed in the final, dried product. This reasoning is in complete agreement with electrospraying experimental observations, where a decrease in particle size with increased voltage has been demonstrated. ${ }^{25,48}$ Under all circumstances, the dynamics in the good solvent THF - allows polymers to form continuously bridged structures, which lead to micron sized particles after drying. Thus, at a fixed voltage and feed rate, we expect larger mesoparticles to form as polymer concentration is increased. This is in accord with our previous experimental results (see Fig. 1 in ref. 24) and also with the observations of other research groups. ${ }^{25,48}$

On the other hand, the polymers form stable nanoscopic aggregates in DMF (Fig. 6b), which are surrounded by solvent molecules (Fig. 8b, inset). These structures are conserved even under high shear rates (Fig. 6) and probably also during electrodriven flow. DMF is much less volatile (boiling point is $146{ }^{\circ} \mathrm{C}$, 
relative evaporation rate (butyl acetate $=1$ ) is 0.2 ) and has low viscosity $\left(3.8 \times 10^{-3}\right.$ Pa s $),{ }^{18}$ yielding low $h$ and relatively lower $D$ in return. Thus, there is again competition between boundary resistance and diffusion resistance to solvent loss, leading to uniform drying. Moreover, polymer clusters keep their isolated structures while forming the surface. Therefore, the result is a nanoscopically rough surface (Fig. 1b), since only stable nanoscopic aggregates are left behind.

In the binary solvent system, swollen polymeric aggregates which are THF rich inside and DMF rich on the surface are formed in a dynamical equilibrium (Fig. 8); these, too, are stable under low shear rates (Fig. 6). At initial times, DMF with its lower $h$ will be present at the air interface while THF molecules having slightly higher diffusivity predominantly reside between the polymeric aggregates. Thus, the amount of drying at stages (a) and (b) will be low, making the process diffusion controlled, allowing enough time for the THF molecules to diffuse to the interface. Up to stage (c), THF molecules evaporate from the surface of these droplets, while the much less volatile DMF is trapped in the core. Once the system is relatively DMF rich, the polymeric aggregates observed in pure DMF form from the polymers that have not had enough time to diffuse into the outer layer. DMF also dries out of the system, the nanoscopic scale roughness from the individual aggregates forms on top of the base layer. As a result, due to inhomogeneous drying processes, the solidification step affects the resulting particle morphology, for which the choice of solvent is critical. Perfectly hydrophobic electrosprayed surfaces may be designed by careful manipulation of solvent kinetics in the consecutive drying stages. For instance, dual scale roughness is not observed on the electrosprayed surfaces of poly(acrylonitrile-co-perfluoroalkyl ethylacrylate) from the THF-DMF binary system, where the copolymer leads to a positive excluded volume in DMF since DMF and THF are the good and poor solvents, respectively (data not shown).

\section{Conclusions}

In this work, we have sought the underlying structural features that yield dual scale roughness which in turn leads to perfectly hydrophobic surfaces prepared by electrospraying a designed polymer dissolved in binary mixtures of poor and good solvents. ${ }^{24}$ For this purpose, we have studied the single chain dynamics of relatively short constructs of the polymers in atomistic detail via MD simulations. We have also predicted the multi-chain morphologies using the coarse-grained DPD approach, and we have tested the stability of the phases formed under shear.

Our previous experimental studies clearly demonstrate that nanoscale roughness occurs on the micrometer scale beads as long as the high boiling point liquid in the solution is the poor solvent. ${ }^{24}$ On the other hand, the current study is a first attempt to rationalize the polymer-solvent interactions and resultant electrosprayed polymer morphologies from a computational point of view. Being a coarse-grained approach, the DPD interaction parameters will have similar values under the conditions outlined above and will lead to similar behavior.
Thus, this work prescribes the main conditions that will lead to dual scale roughness obtained by electrospraying. The systems must be prepared in solutions that will allow diffusion controlled drying to occur. Thus, the chemistry of the polymers must be tuned such that the more volatile component adheres to the polymers while the less volatile component is abundant in the bulk of the solvent. Furthermore, processing conditions may also be tuned so that the boundary resistance controlled drying (phases (a) and (b) in the previous section) is not encouraged. In particular, phase (a) may be suppressed by keeping the voltage low or the feed rate high.

\section{Acknowledgements}

Elif Ozden-Yenigun acknowledges the TUBITAK BIDEB graduate student scholarship. We thank Oguzhan Oguz and Aydin Ozcan for their help with DLS experiments.

\section{References}

1 J. G. Wang, G. P. Mao, C. K. Ober and E. J. Kramer, Macromolecules, 1997, 30, 1906-1914.

2 V. C. Malshe and N. S. Sangaj, Prog. Org. Coat., 2005, 53, 207-211.

3 E. Giannetti, J. Fluorine Chem., 2005, 126, 625-632.

4 D. M. Lemal, J. Org. Chem., 2004, 69, 1-11.

5 W. Groh and A. Zimmermann, Macromolecules, 1991, 24, 6660-6663.

6 S. Agarwal, S. Horst and M. Bognitzki, Macromol. Mater. Eng., 2006, 291, 592-601.

7 J. G. Wang and C. K. Ober, Macromolecules, 1997, 30, 75607567.

8 J. Tsibouklis, M. Stone, A. A. Thorpe, P. Graham, T. G. Nevell and R. J. Ewen, Langmuir, 1999, 15, 7076-7079.

9 R. D. V. de Grampel, W. Ming, A. Gildenpfennig, W. J. H. van Gennip, J. Laven, J. W. Niemantsverdriet, H. H. Brongersma, G. de With and R. van der Linde, Langmuir, 2004, 20, 6344-6351.

10 R. A. Segalman, B. McCulloch, S. Kirmayer and J. J. Urban, Macromolecules, 2009, 42, 9205-9216.

11 E. Martinelli, S. Agostini, G. Galli, E. Chiellini, A. Glisenti, M. E. Pettitt, M. E. Callow, J. A. Callow, K. Graf and F. W. Bartels, Langmuir, 2008, 24, 13138-13147.

12 W. Barthlott and C. Neinhuis, Planta, 1997, 202, 1-8.

13 X. J. Liu, Y. M. Liang, F. Zhou and W. M. Liu, Soft Matter, 2012, 8, 2070-2086.

14 B. Bhushan and Y. C. Jung, Prog. Mater. Sci., 2011, 56, 1-108. 15 Z. G. Guo, W. M. Liu and B. L. Su, J. Colloid Interface Sci., 2011, 353, 335-355.

16 Y. Y. Yan, N. Gao and W. Barthlott, Adv. Colloid Interface Sci., 2011, 169, 80-105.

17 K. Acatay, E. Simsek, C. Ow-Yang and Y. Z. Menceloglu, Angew. Chem., Int. Ed., 2004, 43, 5210-5213.

18 J. Liu, A. Rasheed, H. M. Dong, W. W. Carr, M. D. Dadmun and S. Kumar, Macromol. Chem. Phys., 2008, 209, 2390-2398.

19 A. M. Ganan-Calvo, J. Aerosol Sci., 1999, 30, 863-872. 
20 J. S. Kim, W. S. Chung, K. Kim, D. Y. Kim, K. J. Paeng, S. M. Jo and S. Y. Jang, Adv. Funct. Mater., 2010, 20, 3538-3546.

21 T. Fukuda, H. Asaki, T. Asano, K. Takagi, Z. Honda, N. Kamata, J. M. Ju and Y. Yamagata, Thin Solid Films, 2011, 520, 600-605.

22 T. Fukuda, K. Takagi, T. Asano, Z. Honda, N. Kamata, H. Shirai, J. Ju, Y. Yamagata and Y. Tajima, Jpn. J. Appl. Phys., 2012, 51, 02BK12.

23 T. Fukuda, K. Takagi, T. Asano, Z. Honda, N. Kamata, K. Ueno, H. Shirai, J. Ju, Y. Yamagata and Y. Tajima, Phys. Status Solidi RRL, 2011, 5, 229-231.

24 E. Simsek, K. Acatay and Y. Z. Menceloglu, Langmuir, 2012, 28, 14192-14201.

25 C. H. Park and J. Lee, J. Appl. Polym. Sci., 2009, 114, 430-437.

26 H. G. Wang, Q. W. Liu, Q. B. Yang, Y. C. Li, W. Wang, L. Sun, C. Q. Zhang and Y. X. Li, J. Mater. Sci., 2010, 45, 1032-1038.

27 P. Gupta, C. Elkins, T. E. Long and G. L. Wilkes, Polymer, 2005, 46, 4799-4810.

28 G. D. J. Phillies, Macromolecules, 1984, 17, 2050-2055.

29 MATERIAL-STUDIO, San Diego, 2002.

30 H. Sun, J. Phys. Chem. B, 1998, 102, 7338-7364.

31 H. C. Andersen, J. Chem. Phys., 1980, 72, 2384-2393.

32 H. J. C. Berendsen, J. P. M. Postma, W. F. Vangunsteren, A. Dinola and J. R. Haak, J. Chem. Phys., 1984, 81, 3684-3690.

33 G. Kacar, C. Atilgan and A. S. Ozen, J. Phys. Chem. C, 2010, 114, 370-382.
34 J. H. Hildebrand, The Solubility of Non-Electrolytes, Reinhold, New York, 1936.

35 ACD/ChemSketch, Toronto, ON, Canada, 2003.

36 R. D. Groot and P. B. Warren, J. Chem. Phys., 1997, 107, 4423-4435.

37 K. P. Travis, M. Bankhead, K. Good and S. L. Owens, J. Chem. Phys., 2007, 127, 014109.

38 A. W. Lees and S. F. Edwards, J. Phys. C: Solid State Phys., 1972, 5, 1921.

39 C. E. Barnett, J. Phys. Chem., 1942, 46, 69-75.

40 A. S. Ozen, U. Sen and C. Atilgan, J. Chem. Phys., 2006, 124, 064905.

41 H. Can, G. Kacar and C. Atilgan, J. Chem. Phys., 2009, 131, 124701.

$42 \mathrm{~J}$. N. Israelachvili, Intermolecular and surface forces/Jacob $\mathrm{N}$. Israelachvili, Academic Press, London; San Diego, 1991.

43 E. Ozden, Y. Z. Menceloglu and M. Papila, ACS Appl. Mater. Interfaces, 2010, 2, 1788-1793.

44 E. Ozden-Yenigun, Y. Z. Menceloglu and M. Papila, ACS Appl. Mater. Interfaces, 2012, 4, 777-784.

45 K. Bilge, E. Ozden-Yenigun, E. Simsek, Y. Z. Menceloglu and M. Papila, Compos. Sci. Technol., 2012, 72, 1639-1645.

46 C. M. Hansen, The Three Dimensional Solubility Parameter and Solvent Diffusion Coefficient, Danish Technical Press, Copenhagen, 1967.

47 M. Yoshida and H. Miyashita, Chem. Eng. J., 2002, 86, 193-198. 48 G. Kim, J. Park and H. Han, J. Colloid Interface Sci., 2006, 299, 593-598. 